\title{
Identificación y caracterización de plagas y enfermedades asociadas con el cultivo tradicional de piña Ananas comosus (L. Merr) en comunidades del municipio de Lloró, departamento del Chocó, Colombia
}

\section{Identification and characterization of pests and diseases associated traditional cultivation of pineapple Ananas comosus (L. Merr) in the Municipality of Lloró Communities, Department of Chocó, Colombia}

\section{Samia Yisella Mosquera Ramírez*}

\section{Resumen}

Las plagas y enfermedades son un serio problema en la piña, si no se controlan adecuadamente estas puedenocasionar pérdidas tan grandes que las cosechas son afectadas hasta en un $80 \%$ y en casos especiales la pérdida total. Con el objetivo de conocer e identificar las plagas y enfermedades asociadas con el cultivo de piña en 4 comunidades del municipio de Lloró, se realizó un muestreo sistemático que consistió en hacer un recorrido en zigzag dentro de cada parcela experimental (finca) hasta completar $1 \%$ del total de plantas de esa parcela; se realizaron 10 puntos de muestreo dentro de cada una, evaluándose entre 20 y 60 plantas por cada punto de acuerdo con el tamaño de la parcela. Al mismo tiempo se evaluaron las plagas y las enfermedades que afectan el cultivo, dirigiendo el muestreo hacia la raiz, tallo, hojas y frutos. Como resultado se pudo determinar un total de 5 plagas asociadas con el cultivo de piña (picudo Metamasius dimidiatipennis, cochinilla harinosa Dysmicoccus brevipes, la tecla Strymon basilides, el gusano soldado Elaphria nucicolora y la zorra o chucha Eira barbara), con un porcentaje promedio de incidencia de 10,4\% y dos enfermedades (la gomosis o fusariosis de la piña y la WIT o marchitez de la hoja), con un porcentaje de incidencia de 8,3\%. Ambos casos considerados como leve según la escala (0-20\% leve, 20-50\% moderado y $>50 \%$ fuerte), lo que indica que realmente son mínimos las pérdidas y el nivel de daño económico causado por la presencia de estas plagas y enfermedades.

Palabras clave: Cultivos de piña, Enfermedades, Fincas, Plagas, Porcentaje de incidencia.
Investigadora asociada II, Instituto de Investigaciones Ambientales del Pacífico (IIAP) «John Von Neumann», Quibdó, Colombia. e-mail: $\underline{\text { smosquera@iiap.org.co }}$ 
Plagas y enfermedades asociadas con la piña. SY Mosquera Ramírez

\section{Abstract}

Pests and diseases are a serious problem in pineapple, if not properly control these can cause such large losses that crops are affected up to $80 \%$ and in special cases total loss. In order to understand and identify pests and associated with the cultivation of pineapple in communities Township wept disease, a systematic sampling was to make a zigzag path within each experimental plot (farm) to complete the $1 \%$ of was held total plants of that parcel 10 sampling points were performed within each, evaluated 20 to 60 plants per point depending on the size of the parcel; at the same time pests and diseases affecting the crop were evaluated; directing the sampling towards the root, stem, leaves and fruit. As a result, it was determined a total of 5 pests associated with the cultivation of pineapple (weevil Metamasius dimidiatipennis, cochinilla mealy Dysmicoccus brevipes, the Strymon basilides key, the armyworm Elaphria nucicolora and zorra or shit Eira barbara), with an average incidence rate of $10.4 \%$ and 2 disease (fusarium gummosis or pineapple and wilt WIT or leaf), with an incidence rate of $8.3 \%$. In both cases considered mild to scale (0$20 \%$ mild, moderate $20-50 \%$ and $>50 \%$ strong), indicating that actually lost or level of economic damage caused by the presence of these pests and diseases it is minimal.

Keywords: Diseases, Farms, Incidence rate, Pests, Pineapple crops.

\section{Introducción}

La familia Bromeliaceae, originaria principalmente de América tropical, desde la Tierra del Fuego en Argentina hasta el sur de los Estados Unidos de América, y una especie en el oeste de África. Se han reportado aproximadamente 3.086 especies organizadas en 56 géneros (Luther
2006), dentro de las cuales se encuentra la piña (Ananas comosus L. Merr.) que partiendo de su domesticación es una especie muy conocida por algunas cualidades relacionadas con su sabor, su fragante aroma, su atractivo color amarillo y sus características nutritivas entre otras; su consumo se difundió rápidamente; es la tercera fruta tropical más importante en el mundo, después del banano y los cítricos (Bartholomew et al. 2003). Las altas productividades del cultivo de piña dependen del éxito que se tenga en el manejo de cada uno de los factores controlables, como el suministro de agua y nutrientes, así como el control de plagas invertebradas y enfermedades.

En el departamento del Chocó existen más de 10 cultivares o ecotipos de piña (cultivados de forma natural sin el uso de insumos agrícolas), los cuales no han sido estudiados genéticamente para definirlos como variedades, pero se pueden diferenciar por sus características organolépticas, morfología y tamaño (Medina et al. 2014). La variedad que más se cultiva en el departamento y en especial los agricultores del municipio de Lloró es la piña de castilla o piña lloroseña, es de buena calidad, por lo general jugosa y dulce; la cáscara es delgada en relación con las otras variedades de piña, presenta ojos profundos y separados, el corazón es muy delgado y no produce molestia ni ardor en la boca al consumirla.

Estos cultivos son atacados por diversas plagas $y$ enfermedades, que ocasionan bajas producciones, lo cual se refleja en la disminución del fruto en los mercados locales, lo que ha producido la utilización de variedades introducidas como la perolera, cayena lisa y manzana.

El Instituto de Investigaciones Ambientales del Pacifico (IIAP), Quibdó, Chocó, en cumplimiento de su rol misional, adelantó actividades encaminadas a conocer e identificar las plagas y enfermedades asociadas con el cultivo de piña que permitan generar información que aporten al conocimiento de la especie, además de ser un insumo básico para la construcción de alternati- 


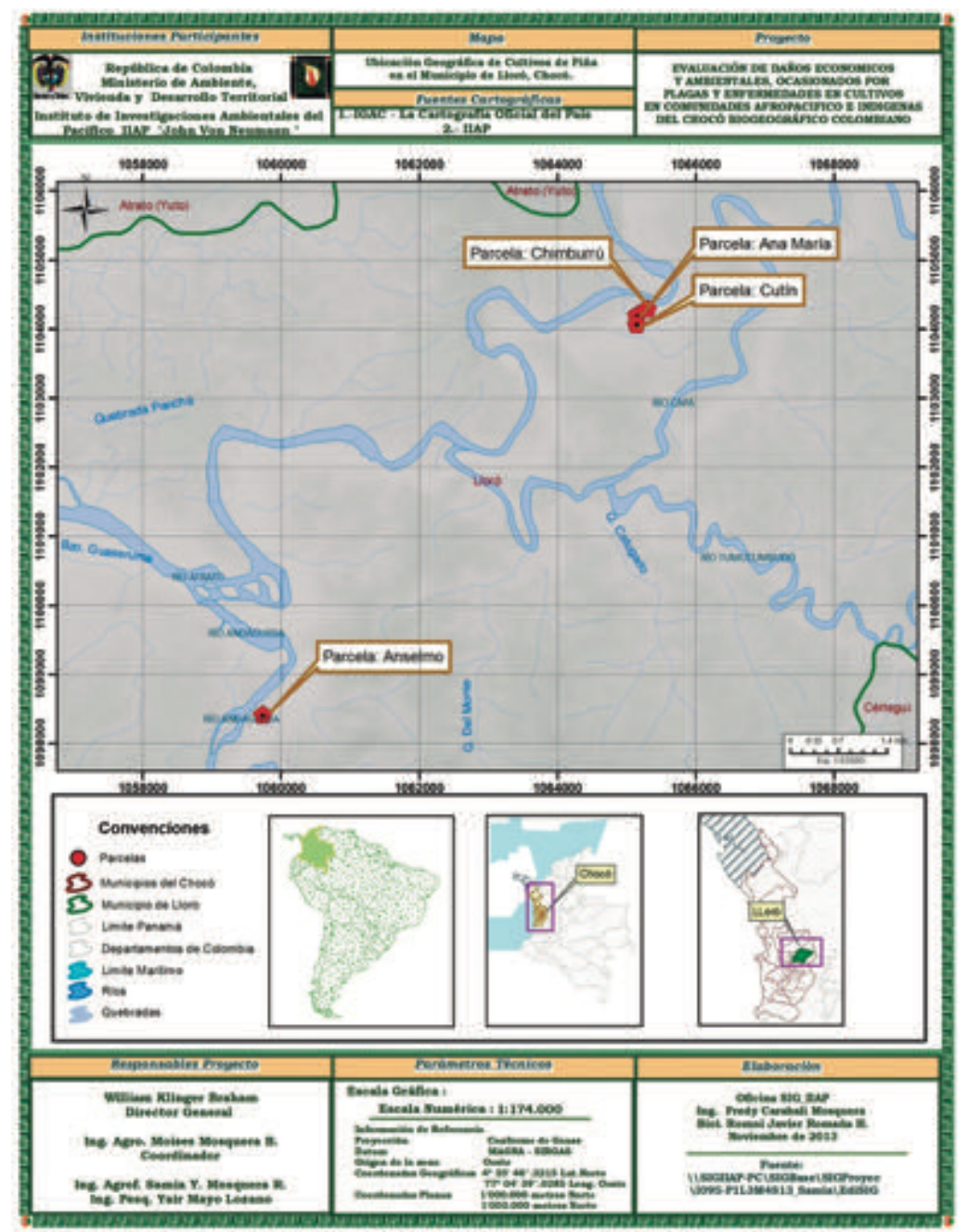

Figura 1. Mapa de ubicación del municipio de Lloró (área de estudio).

vas agroproductivas encaminadas a su manejo integral, que permita optimizar la rentabilidad del cultivo.

Área de estudio. El municipio de Lloró está situado en la zona occidental del departamento del Chocó parte alta del río Atrato a $12 \mathrm{~km}$ del municipio del Atrato; posee una extensión total de $905 \mathrm{~km}^{2}$, los asentamientos de sus pobladores se distribuyen a lo largo de cuatro ríos: el Atrato, Andagueda, Capa y Tumutumbudo (Figura 1). Limita al norte el municipio de Quibdó y El Carmen de Atrato, al sur con Tadó y Bagadó, al este con El Carmen de Atrato y Bagadó y al oeste con el municipio de Atrato. Tiene una altura de $65 \mathrm{msnm}$ y una temperatura promedio de $28^{\circ} \mathrm{C}$
(Alcaldía Municipal de Lloró 2012).

La red hidrográfica del municipio de Lloró se ubica en la cuenca del río Atrato, con las subcuencas de los ríos Capa y Andagueda. Esta red es amplia y de textura fina. El municipio de Lloró y las zonas circunvecinas constituyen el núcleo de máxima precipitación pluviométrica en el territorio colombiano, destacándose por ser una de las regiones más húmedas de la América tropical y una da las más lluviosas del mundo. Estas condiciones de humedad casi permanente, unida a la alta nubosidad y a los cambios drásticos de temperatura, hacen del clima uno de los factores predominantes de la morfología, característica que exhiben los suelos y al mismo tiempo constituyen 
un factor limitante en el uso de la tierra, restringiendo considerablemente el potencial agrícola de la región (Alcaldía Municipal de Lloró 2012).

De acuerdo con el sistema de clasificación para Colombia, la zona presenta un clima cálido muy húmedo, hace parte del Bacín del Atrato y está localizado sobre el sistema de colinas que varían entre 50 y $100 \mathrm{~m}$. Su temperatura promedia es de $26^{\circ} \mathrm{C}$. En este municipio se presentan tres unidades climáticas: medio super húmedo, equivalente a un área de $9,8 \mathrm{~km}^{2}$, clima medio húmedo $325 \mathrm{~km}^{2}$, equivalente a $36 \%$ y una región cálida super húmeda con $562.4 \mathrm{~km}^{2}$, correspondiente a $63 \%$ del territorio. En toda el área la precipitación pluvial promedio anual es mayor de $7200 \mathrm{~mm}$ (Alcaldía Municipal de Lloró 2012).

Las actividades económicas de Lloró corresponden principalmente al sector primario de la economía, sobresale la agricultura, la minería y la explotación forestal, es decir, este municipio depende exclusivamente del aprovechamiento de los recursos naturales en forma artesanal.

La minería como actividad económica rural se desarrolla en forma artesanal; últimamente la actividad minera se ha ido reduciendo y las actividades se están centrando en agroforestería, combinando especies maderables con productos como plátano, caña de azúcar, borojó, piña, chontaduro, en extensiones que son para autoconsumo y pocos excedentes son trasladados a los mercados de Quibdó, Atrato, e Istmina. Problemas como falta de asistencia técnica agropecuaria, crédito, vías de acceso, canales de comercialización, e infraestructura adecuada tienen el sector productivo en mal estado (Alcaldía Municipal de Lloró 2012).

El estudio se realizó específicamente en las comunidades de Chimburrú, situada en la margen derecha del río Atrato, se encuentra aproximadamente a 60 minutos por vía fluvial de la cabecera municipal de Lloró, con coordenadas geográficas de $05^{\circ} 32$ ' 30.1" de latitud norte y $076^{\circ} 29$ ' 29.7'" de longitud oeste con Boraudo, La Vuelta y la cabecera municipal.

\section{Método}

Para el desarrollo de la presente investigación se realizó el siguiente diseño metodológico:

Acopio y análisis de la información primariao fase de campo. Se utilizó el método propuesto por Fals-Borda y Rodríguez Brandao (1987) modificada por el equipo de investigación de campo, el cual se basa en la investigación-acción-participación (IAP), para la caracterización e identificación de plagas y enfermedades del cultivo; se realizaron reuniones de socialización y se aplicaron encuestas y entrevistas semiestructuradas a los productores.

Muestreo o identificación de plagas y enfermedades. Para las inspecciones fitosanitarias en campo, se determinó, el diseño de muestreo el cual fue sistemático. Los muestreos para identificar las plagas y enfermedades se realizaron en forma de zigzag dentro de cada parcela experimental (finca) hasta completar 1\% del total de plantas (Figura 2); se realizaron 10 puntos de muestreo dentro de cada una, evaluándose de 20 a 60 plantas por cada punto de acuerdo con el tamaño de la parcela de manera que incluyera toda el área seleccionada para evaluar.

Los muestreos en las parcelas o cultivos de piña se realizaron evaluando al mismo tiempo las plagas y las enfermedades que afectan el cultivo, dirigiendo el muestreo en la etapa de desarrollo de plantas hacia la raíz y la planta, y en la etapa

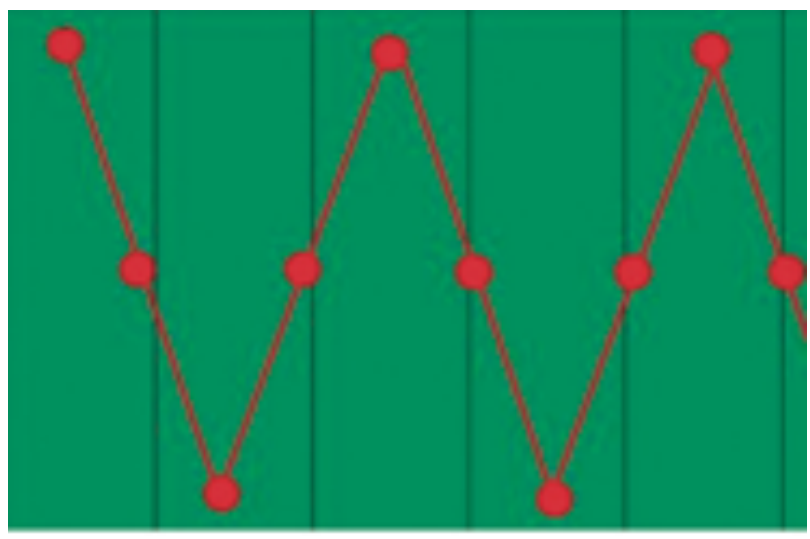

Figura 2. Diseño sistemático del muestreo dentro de una sección de la parcela (finca). 


\section{Bioetnia Volumen 13, 2016}

de desarrollo de fruta, hacia la fruta. Se realizaron muestreos en las raíces de las plantas, hojas y frutos con el ánimo de identificar la presencia de plagas y enfermedades en los cultivos. Para determinar la presencia de estas plagas se realizó una búsqueda exhaustiva y conteo por planta en la base del fruto, entre las hojas ubicadas en la unión del pedúnculo. Además, se sacrificaron algunas frutas para hacerles el muestreo interno.

La incidencia se evaluó como la cantidad de plantas afectadas por la plaga, para lo cual se aplicó la ecuación:

$$
\mathrm{GI}=(\mathrm{NPA} / \mathrm{NTP}) * 100
$$

donde:

$\mathrm{GI}=$ Grado de incidencia

NPA $=$ Número de plantas afectadas

$\mathrm{NTP}=$ Número total de plantas

\section{Resultados y discusión}

Caracterización e identificación de plagas. Aunque son muchos los insectos dañinos que han sido identificados como asociados al cultivo de piña, no todos se consideran plagas de importancia económica, solo algunos de ellos afectan el rendimiento del cultivo, la calidad de la fruta, requiriendo algún tipo de control. En el municipio de Lloró se identificó una menor cantidad de insectos perjudiciales presentes en el cultivo de piña con niveles de incidencia no muy elevados. Se registraron 5 especies de insectos plagas representadas en 5 familias y 5 géneros, de las cuales 2 pertenecen al orden Lepidoptero tecla (Strymon basilides) y gusano soldado (Elaphria nucicolora). Del total de las especies encontradas, las comunidades de Boraudo y cabecera municipal registraron presencia de las 5 especies, seguida por las comunidades de Chimburrú y La Vuelta con 4 del total de las especies reportadas.

Por otro lado, de las 390 plantas inspeccionadas se registró una afectación por plagas de 202 plantas en los diferentes cultivos evaluados, de las cuales 68 pertenecen a la comunidad de Boraudo, seguida de Chimburrú con 64, la cabecera municipal con 39 y La Vuelta con 31 (Figura 3).

Estas especies identificadas se clasifican de acuerdo con sus hábitos y el daño que ocasionan, como plagas de la raíz, el tallo, las hojas y el fruto. Entre los insectos dañinos presentes en el área de estudio por la severidad de sus daños se encuentra la cochinilla harinosa Dysmicoccus brevipes, el picudo Metamasius dimidiatipennis, que ataca directamente frutos, tallos y raíz, la tecla Strymon basilides, el gusano soldado Elaphria nucicolora y la zorra o chucha Eira barbara que ataca el fruto cuando está maduro (Tabla 1).

Las pérdidas ocasionadas por plagas en los cultivos de piña en el municipio no son considerables. Afectan algunas especies de insectos, tanto de forma directa, como por ser vectores de

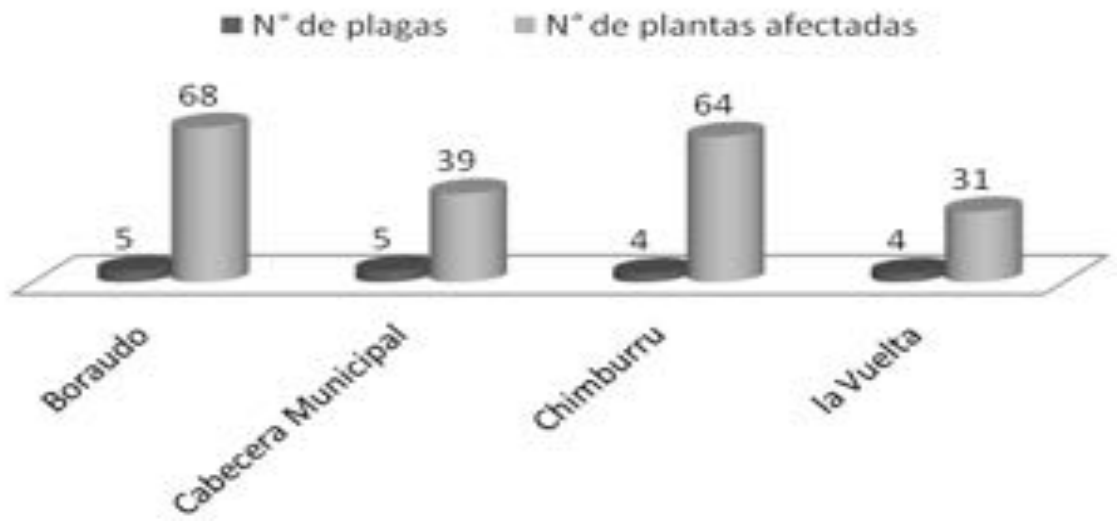

Figura 3. Número de plantas afectadas por plagas y en las diferentes comunidades evaluadas. 
Plagas y enfermedades asociadas con la piña. SY Mosquera Ramírez

Tabla 1. Insectos/plagas identificadas en las comunidades del municipio de Lloró

\begin{tabular}{|c|c|c|c|}
\hline Insecto/plagas & Nombre científico & Daño & $\%$ de incidencia \\
\hline $\begin{array}{l}\text { Cochinilla } \\
\text { harinosa }\end{array}$ & $\begin{array}{l}\text { Dysmicoccus } \\
\text { brevipes }\end{array}$ & Succionan savia de raíces y tallos & Leve - 23,3 \\
\hline Picudo & $\begin{array}{l}\text { Metamasius } \\
\text { dimidiatipennis }\end{array}$ & $\begin{array}{l}\text { Tallo y raíces de la planta; sin embargo, también } \\
\text { afecta el pedúnculo, hijos y fruto. }\end{array}$ & Leve $-3,8$ \\
\hline Gusano soldado & Elaphria nucicolora & $\begin{array}{l}\text { La larva ocasiona un raspado superficial de la } \\
\text { fruta, produciendo una coloración translucida de } \\
\text { la pulpa y a menudo una gomosis externa. }\end{array}$ & \\
\hline Tecla & Strymon basilides & $\begin{array}{l}\text { Ocasiona galerías internas en la pulpa, produ- } \\
\text { ciendo un exudado conocido como gomosis en } \\
\text { la parte externa de la fruta. }\end{array}$ & Leve - 3,3 \\
\hline \multirow[t]{2}{*}{ Roedores (zorra) } & Eira barbara & Se come el fruto en estado de madurez. & Leve - 8,5 \\
\hline & & Total (Promedio) & 10,4 \\
\hline
\end{tabular}

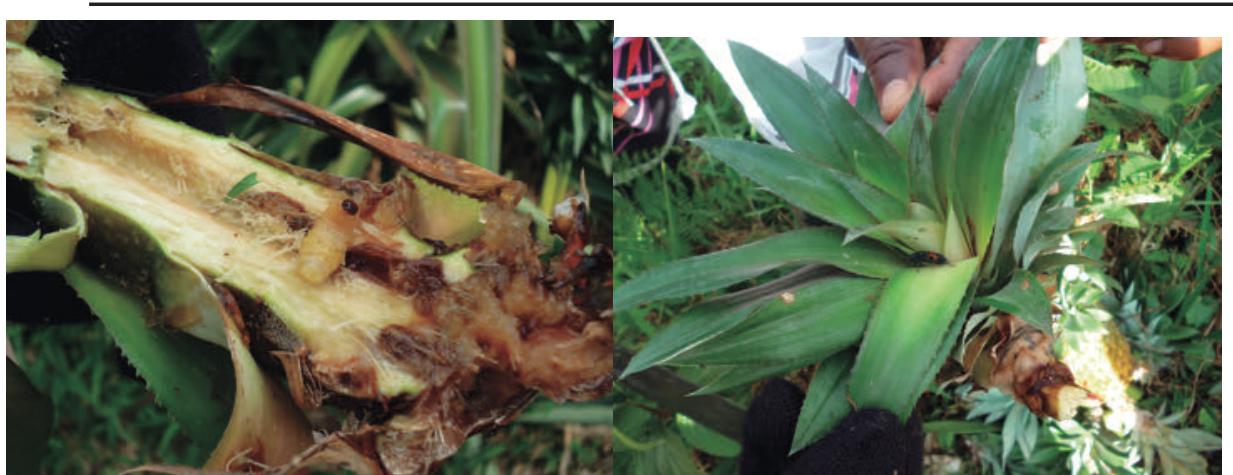

Figura 4. Presencia de larva y adulto de picudo.

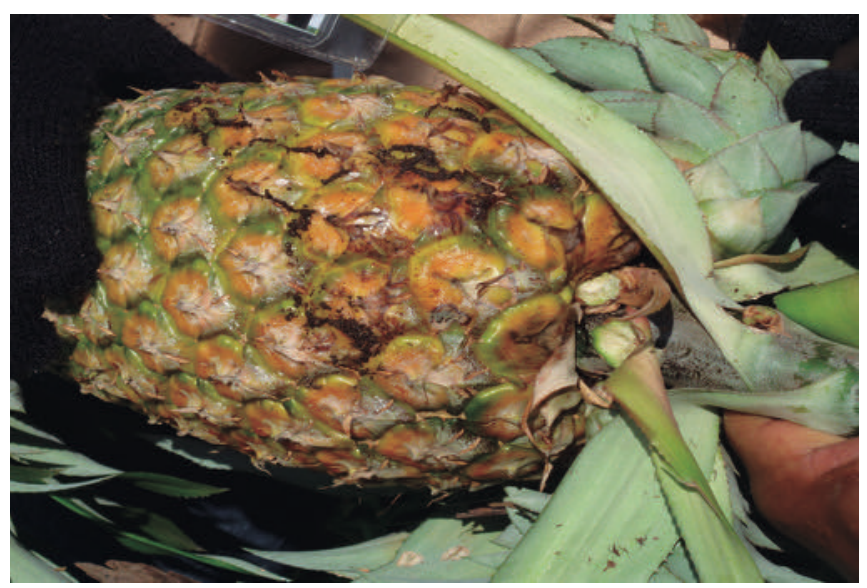

Figura 5. Fruto afectado por la cochinilla harinosa (Dysmicoccus brevipes).

enfermedades como la cochinilla harinosa, el gusano barrenador del fruto (tecla) Strymon basilides, la zorra y otros agentes que constituyen un peligro potencial. La cochinilla harinosa es el agente de mayor importancia, tanto por el daño directo como por ser el vector del virus de la marchitez.

Los resultados muestran que de las 5 plagas encontradas, el picudo Metamasius dimidiatipennis (Figura 4), del orden Coleoptera y familia Curculionidae, es el insecto con mayor presencia, encontrándose en las cuatro comunidades y en las 12 fincas o parcelas visitadas, con un total de 91 plantas afectadas, siendo la comunidad de Boraudo la de mayor incidencia con 35 plantas, seguida de la comunidad de Chimburrú con 25, la cabecera municipal con 21 y La Vuelta con 10, dato que podría estar relacionado con los períodos de lluvia que presenta la zona porque según De Bravo (1997) afirma que este se propaga con mayor facilidad en los períodos húmedos.

En segundo lugar, se encuentra la cochinilla harinosa (Dysmicoccus brevipes), es un insecto pequeño (Figura 5), de color blanco que se ubica en las axilas de las hojas inferiores de la planta, raíces y el fruto. Su presencia es durante todo el año según datos suministrados por los productores visitados, con mayor intensidad 


\section{Bioetnia Volumen 13, 2016}

durante los períodos de floración, fructificación, postcosecha, estado vegetativo y de crecimiento, dato que concuerda con lo expuesto por Ortega y Arauz (2015), donde afirma que esta plaga ataca cualquier parte de la planta durante todo el ciclo del cultivo, aunque en el presente estudio se ubica en el segundo lugar; de acuerdo con el número de plantas afectadas está catalogada por los investigadores (De Bravo1997, Domínguez Barradas 2013, Ortega y Arauz 2015) y productores, como el insecto plaga de mayor importancia en el cultivo de piña; tuvo presente en siete de las 12 fincas visitadas, con un total de 50 plantas afectadas, siendo la comunidad de Chimburrú la que presentó mayor número de plantas afectadas (21), seguida de La Vuelta (15), Boraudo (10) y cabecera municipal (4) plantas afectadas.

La zorra Eira barbara, del órden Carnivora y familia Mustelidae, es otra de las plagas de interés para los productores con una cantidad de 33 plantas afectadas; ella se come el fruto cuando está maduro, afectando el rendimiento de la cosecha; la comunidad de Chimburrú en este caso presentó el mayor grado de afectación con un total de 14 plantas afectadas, seguido de la cabecera municipal con 8, Boraudo con 6 y finalmente La Vuelta con 5 del total de plantas afectadas. Por su parte, el gusano (15) y la tecla (13) fueron las plagas con menor grado de incidencia en el presente estudio (Figura 6).

Nivel de incidencia de las plagas. La Figura 7 muestra, un promedio en el porcentaje de incidencia de plagas en las cuatro comunidades

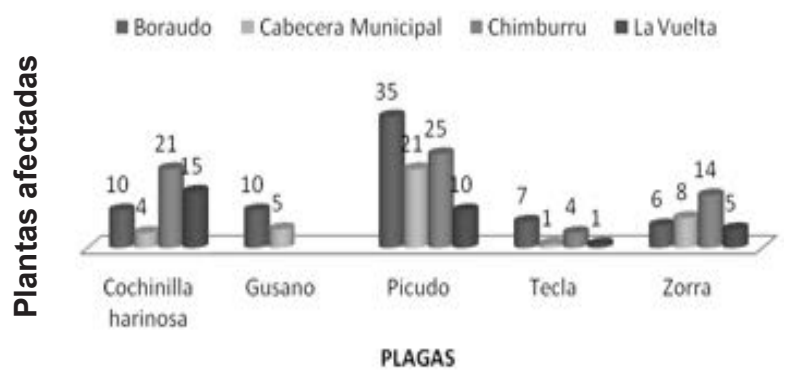

Figura 6. Tipo de plaga y cantidad de plantas afectadas por comunidad. de $10,4 \%$ del total de 390 plantas evaluadas, lo que sería catalogado como leve según Flores et al. (2010) de acuerdo con su tabla (leve $0-25 \%$, moderado $26-50 \%$, fuerte $>50 \%$ ) para determinar la incidencia de los insectos plaga en plantaciones de teca. En ese orden de idea, el nivel más alto lo registró Metamasius dimidiatipennis con 23,3\%, seguido por la Dysmicoccus brevipes $12,8 \%$. Este resultado que podría ser por las intensas jornadas de lluvias que se presentan en la zona considerada como uno de los sitios más lluviosos del mundo y además, esta especie según la literatura se prolifera más rápido en los períodos de humedad. Sin embargo, los niveles más bajos fueron registrados en Strymon basilides y Elaphria nucicolora (3\%).

Caracterización e identificación de enfermedades. La piña, al igual que muchos otros cultivos tropicales, es afectada por enfermedades durante las diferentes fases de su crecimiento, algunas veces restringiendo su producción en ciertas áreas y otras, elevan los costos de producción por efectuarse medidas preventivas o curativas para bajar los niveles de daño.

Los resultados obtenidos muestran que las enfermedades que afectan a los cultivos de piña en las cuatro comunidades objeto de investigación en el municipio de Lloró fueron en orden de importancia la gomosis, que ocasiona la deformación de los frutos, seguido de la marchitez de la hoja causada por el vector Pseudococcus brevipes Ckll trasmitido por la cochinilla harinosa, que provoca un desecamiento del ápice hacia la base de la hoja y un enrollamiento en el borde de las hojas más afectadas (Tabla 2).

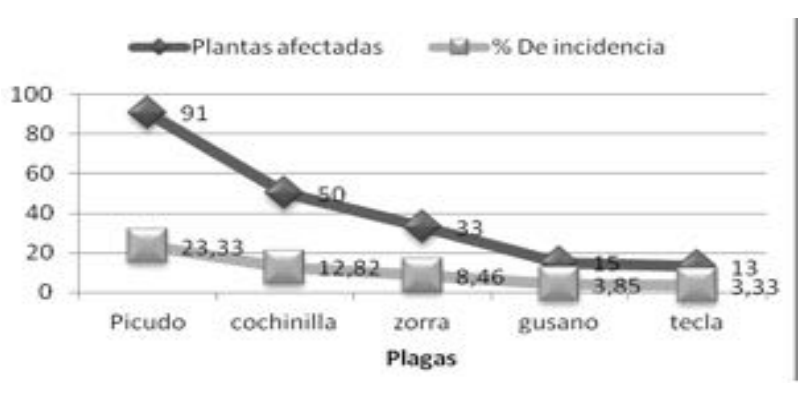

Figura 7. Porcentaje de incidencia de plagas. 
Plagas y enfermedades asociadas con la piña. SY Mosquera Ramírez

Tabla 2. Enfermedades identificadas en comunidades del municipio de Lloró

\begin{tabular}{lccc}
\hline \multicolumn{1}{c}{ Enfermedades } & Nombre científico & Daño & \% de incidencia \\
\hline $\begin{array}{l}\text { Gomosis, agente causal } \\
\text { Marchitez de las hojas, }\end{array}$ & Fusariun moniliforme var subglutinans & Frutos y semillas & Leve $-14,6$ \\
$\begin{array}{l}\text { agente causal } \\
\text { Total (Promedio) }\end{array}$ & Pseudococcus brevipes Ckll & Hojas & Leve - 2,1 \\
\hline
\end{tabular}

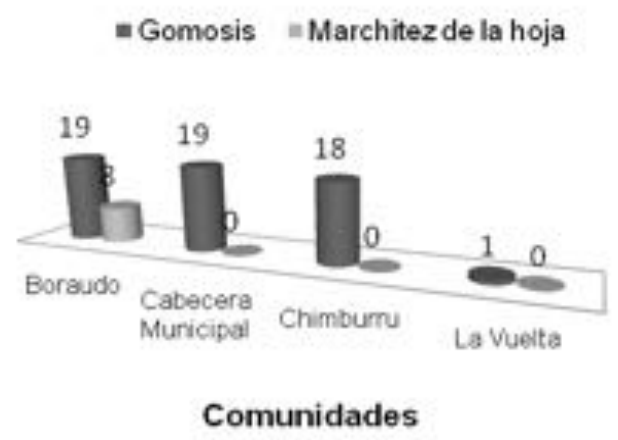

Figura 8. Tipo de enfermedad y cantidad de plantas afectadas por comunidad.

Los niveles de los daños no fueron elevados, porque de las 390 plantas inspeccionadas solo 65 fueron afectadas por enfermedades, siendo la gomosis la que presentó el mayor número de plantas afectadas (57), siendo la comunidad de Boraudo la más afectada, seguida de la cabecera municipal con 19 cada una y la comunidad de Chimburrú con 18 plantas afectadas. La que presentó menor número de plantas afectadas fue la comunidad de La Vuelta (1). En segundo orden esta la marchitez de la hoja con solo 8 plantas afectadas en la comunidad de Boraudo y solo en dos de las tres fincas visitadas (Figura 8).

Nivel de incidencia. La Figura 9 muestra, un promedio en el porcentaje de incidencia de enfermedades en las cuatro comunidades de 8,3\% del total de 390 plantas evaluadas. Lo que sería catalogado como leve según Flores et al. (2010) de acuerdo con su tabla (leve $0-25 \%$, moderado $26-50 \%$, fuerte $>50 \%$ ) para determinar la incidencia de los insectos plaga en plantaciones de teca. En ese orden de idea, el nivel más alto lo presentó la gomosis o fusariosis de la piña, causada

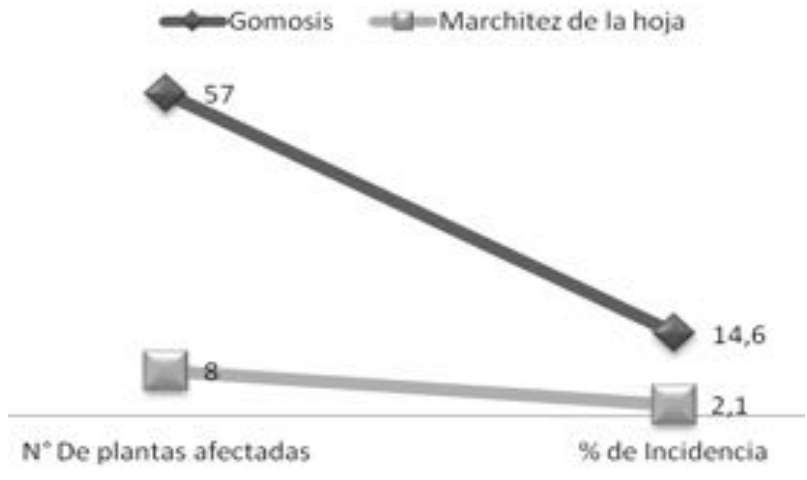

Figura 9. Porcentaje de incidencia de enfermedades.

por Fusarium moliniforme sp., con 14,6\%; este resultado podría ser por la presencia del picudo, del gusano soldado y la tecla porque estos insectos hacen perforaciones que generan un exudado ocasionando la anomalía conocida comúnmente como gomosis de la piña (Figura 10). Por otro lado, la marchitez de la hoja presentó un porcentaje de incidencia $(2,1 \%)$ relativamente bajo, si se hace la relación entre la cantidad de plantas afectadas por la cochinilla harinosa, principal agente trasmisor de la enfermedad y en este estudio se registró como el segundo insecto plaga más importante de acuerdo con el nivel de su daño, por lo que se esperaría un nivel de incidencia mucho más alto.

\section{Conclusiones}

Se registró un total de cinco insectos plagas (la cochinilla harinosa, el picudo, el gusano soldado, la tecla y el roedor la zorra) presentes en los cultivos de piña evaluados en las cuatro comunidades, de las cuales el picudo presentó el mayor número de plantas afectadas (91), además de encontrarse 


\section{Bioetnia Volumen 13, 2016}

en todas las comunidades y las 12 fincas o parcelas visitadas. En segundo lugar, estuvo la cochinilla harinosa con 50 plantas afectadas. El resto de las plagas se presentaron en menor cantidad. El porcentaje de incidencia por el ataque de estas plagas fue considerado como leve $(10,4 \%)$, es decir, que no representa una amenaza relevante en cuanto al rendimiento de las cosechas, pero aunque el nivel no fue representativo amerita que se haga un buen manejo y control a tiempo porque las condiciones tradicionales en las que los agricultores de esta zona realizan las actividades de establecimiento y manejo del cultivo, no cumple con las condiciones técnicas de una buena producción de piña.

Se registró un total de dos enfermedades (la gomosis y la marchitez de las hojas), con un porcentaje de incidencia del ataque de $8,3 \%$, considerado como leve, lo que indica que realmente las pérdidas o el nivel de daño económico causado por ellas es mínimo, por lo tanto se debe aplicar un control manual sin la utilización de ningún químico que ocasione problemas ambientales por su aplicación.

El estudio también demuestra que la producción de piña se realiza principalmente bajo condiciones de tipo artesanal, en predios muy pequeños y donde los cultivos de piñas ocupan una baja extensión en las parcelas. Siendo en la mayoría de los casos de un cuarterón a una hectárea. E1 desconocimiento de las técnicas del cultivo tanto en la producción y las distancias de siembra no son las apropiadas hacen que el establecimiento del cultivo no sea el adecuado limitando así el nivel de producción.

\section{Recomendación}

Los resultados de esta investigación en general no presentaron niveles de daños elevados ni por el ataque de plagas ni enfermedades, por lo que se recomienda realizar prácticas culturales adecuadas para el normal desarrollo del cultivo, tales como: desinfección de los colinos o semillas antes de la siembra, control de arvenses y fertilizaciones periódicas con fertilizantes autorizados en el mercado para tal fin, todo esto para poder garantizar una buena producción.

\section{Literatura citada}

Alcaldía Municipal de Lloró, Chocó. 2012. Plan de Desarrollo Municipal 2012-2015.

Bartholomew DP, Paull RE, Rohrbach KG. 2003. The pineapple: botany, production and uses. New York: CAB International; $301 \mathrm{pp}$.

De Bravo I.M. 1997. El cultivo de la piña en Venezuela. Caracas: Instituto Interamericano de Cooperación para la Agricultura (IICA) y Biblioteca Venezuela; 155 pp.

Domínguez Barradas D. 2013. Potencial del cultivo de piña MD-2 Ananas comosus en el municipio de Juan Rodríguez Clara Veracruz. Zalapa Enríquez: Universidad Veracruzana; 59 pp. URL disponible en: https://es.scribd. com/document/284076686/Dominguez-Barradas

Fals-Borda O, Rodríguez Brandao C. 1987. Investigación participativa. Montevideo: Instituto del Hombre, Ediciones de la Banda Oriental.

Flores T, Crespo R, Cabezas F. 2010. Plagas y enfermedades en plantaciones de teca (Tectona grandis LF) en la zona de Balzar, provincia del Guayas. Ciencia y Tecnología. 3 (1): 15-22. URL disponible en: https:// webcache.googleusercontent.com/search?q=cache:drVEyoncZlUJ:https://dialnet.unirioja.es/descarga/ articulo/4130581.pdf $+\& \mathrm{~cd}=1 \& \mathrm{hl}=\mathrm{es} \& \mathrm{ct}=\mathrm{clnk} \& \mathrm{gl}=\mathrm{co}$

Luther HE. 2006. An alphabetical list of bromeliad binomials. $7^{\text {th }}$ ed. Sarasota: The Marie Selby Botanical Gardens, Bromeliad Society International; 124 pp. URL disponible en: https://selby.org/wp-content/uploads/ Bromeliad_Binomial_List_For_Web.pdf

Medina MA, Mosquera HR, Aguilar CL. 2014. Micropropagación clonal y enraizamiento ex vitro de tres cultivares de piña Ananas comosus (L. Merr.) delChocó, Colombia. Rev Biodivers Neotrop. 4 (2): 133-40. URL disponible en: http://revistas.utch.edu.co/ojs5/index. php/Bioneotropical/article/view/199/117

Ortega EE, Arauz A. 2015. Plagas de importancia durante las etapas fenológicas del cultivo de la piña (Ananas comosus). David: Escuela de Ciencias Agroambientales; 71 pp. URL disponible en: http://www.academia. edu/19590672/PLAGAS DE IMPORTANCIA DURANTE_LAS_ETAPAS_FENOL\%C3\%93GICAS DEL CULTIVO DE LA PI\%C3\%91A Ananas comosus 